Document downloaded from:

http://hdl.handle.net/10251/83592

This paper must be cited as:

Algaba-Brazález, A.; Flygare, J.; Yang, J.; Vassilev, V.; Baquero Escudero, M.; Kildal, P. (2016). Design of F-Band Transition From Microstrip to Ridge Gap Waveguide Including Monte Carlo Assembly Tolerance Analysis. IEEE Transactions on Microwave Theory and Techniques. 64(4):1245-1254. doi:10.1109/TMTT.2016.2535334.

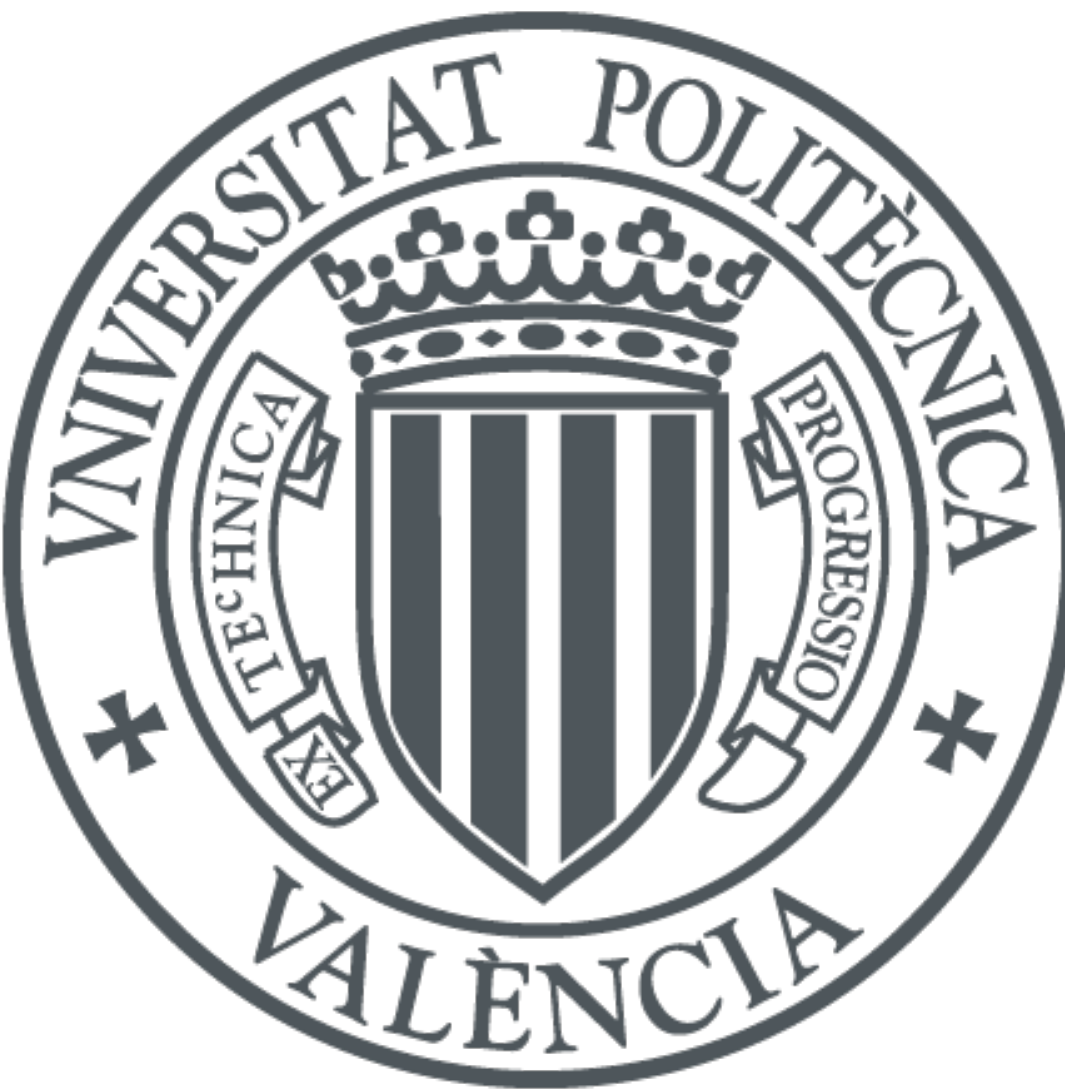

The final publication is available at

http://dx.doi.org/10.1109/TMTT.2016.2535334

Copyright Institute of Electrical and Electronics Engineers (IEEE)

Additional Information

(c) 2016 IEEE. Personal use of this material is permitted. Permission from IEEE must be obtained for all other users, including reprinting/ republishing this material for advertising or promotional purposes, creating new collective works for resale or redistribution to servers or lists, or reuse of any copyrighted components of this work in other works. 


\title{
Design of F-band Transition from Microstrip to Ridge Gap Waveguide including Monte Carlo Assembly Tolerance Analysis
}

\author{
Astrid Algaba Brazález, Jonas Flygare, Jian Yang, Senior Member, IEEE, Vessen Vassilev, Mariano \\ Baquero-Escudero, Member, IEEE and Per-Simon Kildal, Fellow, IEEE
}

\begin{abstract}
This paper describes the design and realization of a transition from a microstrip line to a ridge gap waveguide operating between 95 and 115 GHz. The study includes simulations, measurements, and a Monte Carlo analysis of the assembly tolerances. The purpose of this tolerance study is to identify the most critical misalignments that affect the circuit performance, and to provide guidelines about the assembly tolerance requirements for the proposed transition design.
\end{abstract}

Keywords-Artificial Magnetic Conductor, assembly, F-band, microstrip, millimeter-waves, Monte Carlo, tolerance, transition, ridge gap waveguide.

\section{INTRODUCTION}

$\mathbf{T}$ He fast development that wireless communication is currently experiencing and the need to fulfill higher capacity demands have motivated the urge to investigate new low loss technologies that can replace conventional microstrips, coplanar waveguides (CPW), striplines and standard waveguides at high frequencies. High functionality, performance and integration of active and passive components in minimal volumes set a big challenge for emerging millimeter-wave (mm-wave) technologies. There exists a need of three-dimensional vertically integrated circuits to constitute low volume RF modules [1], but traditional technologies such as microstrip and CPW experience a clear degradation in performance as the frequency increases due to presence of surface waves, radiation problems and other parasitic coupling issues. Standard waveguides do not constitute a cost-effective and easy-to-integrate approach for large corporate feed networks of array antennas at mmwaves because it is very difficult to have a good metal contact between the upper and lower blocks, where the E-plane splitblock waveguide technology [2] cannot be used.

The recently introduced gap waveguide in [3] and [4] constitutes a new approach for mm-wave frequency ranges

\footnotetext{
Manuscript received May 22, 2015.

This work was supported by: the Swedish Research Council VR, the Swedish Governmental Agency for Innovation Systems VINNOVA via a project within the VINN Excellence center Chase, and the European Research Council (ERC) via the advanced investigator grant ERC-2012-ADG20120216.

A. A. Brazález, J. Yang and P.-S. Kildal are with the Department of Signals and Systems, J. Flygare is with Onsala Space Observatory and V. Vassilev is with the Department of Microtechnology and Nanoscience (MC2), all at Chalmers University of Technology, SE-41296 Gothenburg, Sweden.

M. Baquero-Escudero is with the Instituto de Telecomunicaciones y Aplicaciones Multimedia (ITEAM), Universidad Politécnica de Valencia (UPV), 46022 Valencia, Spain.
}

since it is able to overcome those critical issues of traditional technologies. Gap waveguides are based on the field cut-off established by two parallel Perfect Electric Conductor (PEC) and Perfect Magnetic Conductor (PMC) layers which are separated by an air-gap smaller than quarter wavelength. Any electromagnetic wave is forbidden to propagate between these two layers, with the exception of local waves that can follow strips, ridges or grooves embedded within the parallel PECPMC. The PMC condition can be obtained by an Artificial Magnetic Conductor (AMC) in the form of a periodic surface, and together with a PEC layer ensures the removal of any surface waves and parallel-plate modes. The frequency band in which this cutoff is effective is called stopband and has been thoroughly investigated in [5] by applying different types of AMC. The cutoff principle ensures a greater application potential of the gap waveguide than standard waveguides and microstrip lines at mm-waves. First, there is no requirement for good mechanical contact between the metal blocks composing the gap waveguide circuits. Second, this technology has lower loss than microstrip lines since no dielectric material is needed [6]. Third, the surface waves that microstrip lines usually suffer from are efficiently suppressed by the gap waveguide. Moreover, gap waveguide has been also applied as a suitable technique for suppressing radiation from microstrip passive components and increase the isolation between microwave modules [7] - [9], i.e., as a packaging structure.

A simple transition from ridge gap waveguide to coaxial connector was developed and presented in [10] in order to allow measurements of a $\mathrm{Ku}$-band ridge gap waveguide demonstrator described in [11]. This type of transition has been employed in most of our ridge and groove gap waveguide prototypes operating at microwave frequencies. However, new versions of gap waveguide transitions are required as the frequency increases. Several suitable transitions working at 60 $\mathrm{GHz}$ have been already studied and experimentally validated [12], [13]. In addition, some investigations on transitions between a ridge gap waveguide and standard rectangular waveguides at $0.3 \mathrm{THz}$ have been also initiated. Difficulties to perfectly align the ridge gap circuit and the waveguide flange via an inline transition designed by means of steps, which significantly degraded the measurement results, motivated the need of identifying other transition alternatives. Thereby, we decided to investigate transitions from planar structures (coplanar waveguide [14] and microstrip [15]) to a ridge gap waveguide operating in F-band (90-140 GHz). The 
reason to choose this frequency band is that we have available "on wafer" probe stations operating up to $125 \mathrm{GHz}$, which allows us to test those transition designs.

As mentioned, any misalignment between the blocks composing a mm-wave circuit can cause a serious perturbation on its performance. Due to the small wavelengths at frequencies above $100 \mathrm{GHz}$, the tolerance requirements on manufacturing and assembly are stricter than at lower frequencies. Therefore, it is important to know the effect of tolerances on the performance of mm-wave devices in order to impose a proper tolerance requirement. The assembly of a gap waveguide circuit involves an unavoidable human factor that makes this process extremely critical in terms of tolerances. This fact motivates the tolerance study presented in this paper, which is based on a Monte Carlo analysis applied on the designed transition. The cumulative distribution function (CDF) of a random combination of different assembly misalignments for the reflection coefficient of the transition has been extracted. This provides a good guideline for imposing the assembly requirement.

Sections II and III of this paper deal with the design of an F-band transition between microstrip and ridge gap waveguide. This design is a continuation of a preliminary one introduced in [15], which has been suitably improved now. Sections IV and $\mathrm{V}$ focus on the Monte Carlo assembly tolerance analysis to identify the most critical assembly misalignments.

\section{GEOMETRY AND DESIGN OF F-BAND MICROSTRIP TO RIDGE GAP WAVEGUIDE TRANSITION}

A transition design between microstrip and ridge gap waveguide based on mechanical pressure contact was reported in [16], with measured return loss of $14 \mathrm{~dB}$ over 55\% relative bandwidth at Ka-band. The pressure contact approach becomes quite complex to be realized at frequencies above $100 \mathrm{GHz}$ because the height of the air gap in the gap waveguide must be kept equal to the thickness of the substrate. It is also difficult to control the pressure contact area due to the small dimensions of our components at mm-wave frequencies. This motivated the investigation of a new transition approach.

The first step in the design of gap waveguide components is to determine the dimensions of the pin surface to ensure the stopband that covers the operating frequency band. The dimensions of our gap waveguide geometry as well as its dispersion diagram are shown in Fig. 1(a) and Fig. 1(b), respectively. The dispersion diagram was simulated by using CST Eigenmode solver and considering periodic boundary conditions in both $\mathrm{X}$ and $\mathrm{Z}$ directions. The obtained stopband covers the range of frequencies between 70 and $135.8 \mathrm{GHz}$ where only a quasi-TEM mode is allowed to propagate along the path settled by the ridge.

In our transition design, the transformation of the quasiTEM mode of a microstrip line into the quasi-TEM mode of the ridge gap waveguide is obtained via electromagnetic field coupling. This interconnection approach was introduced in [17] and [18], for the design of mm-wave transitions between microstrip lines and coplanar waveguides and proposed as an alternative to bond-wires when interconnecting Millimeterwave Monolithic Integrated Circuits (MMICs) to a carrier

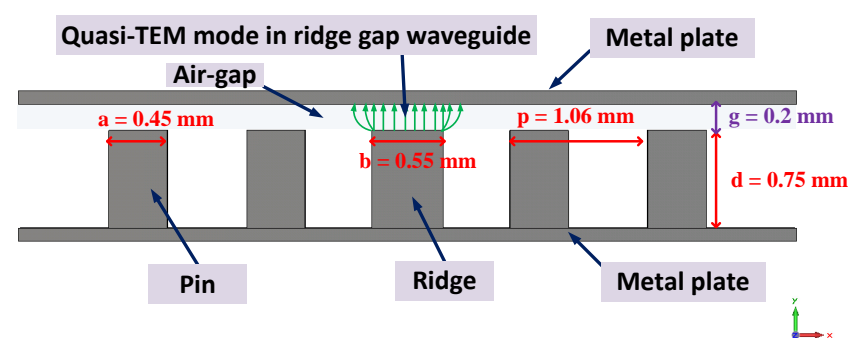

(a)

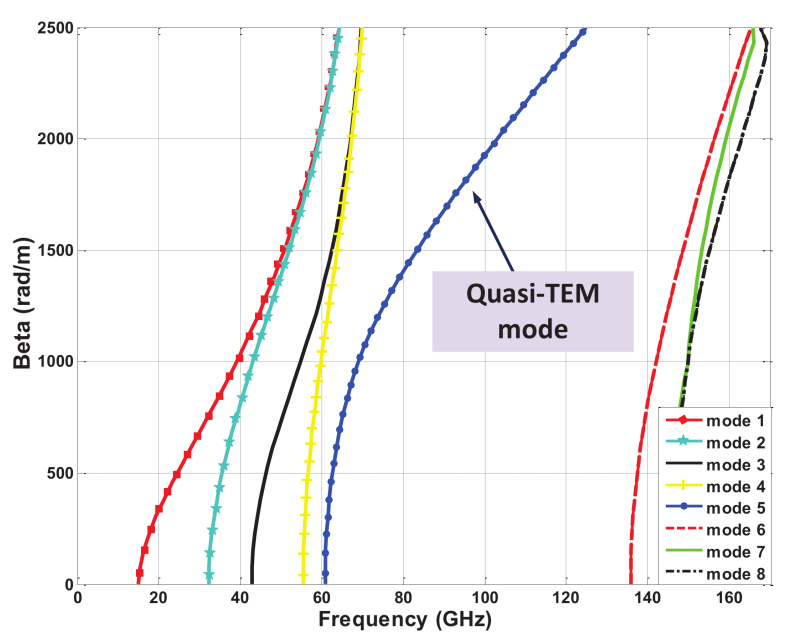

(b)

Fig. 1. (a) Dimensions of ridge gap waveguide. (b) Resulting dispersion diagram.

substrate. This coupling principle consists of overlapping quarter-wavelength transmission line segments, where matching elements can be also included to improve the transition performance. Another successful employment of this interconnection method is presented in [19], where a vertical CPW-tomicrostrip transition operating in W-band shows wideband and low loss performance.

Our F-band transition composed of two layers is ilustrated in Fig. 2. The first layer is a metal plate that contains the ridge gap waveguide interface. The second layer is a Printed Circuit Board (PCB) that includes a microstrip circuit, and the ground plane of the PCB is soldered into the bottom metal plate. The employed substrate is alumina with thickness $h=0.127 \mathrm{~mm}$, dielectric constant $\epsilon_{r}=9.8$ and loss tangent $\tan \delta=0.0002$ (evaluated at $10 \mathrm{GHz}$ according to the material supplier). As shown in Fig. 2, the ridge gap waveguide is placed upside down in the upper metal plate, whilst the PCB positioned in the bottom metal layer is facing upwards towards the ridge. All metallic parts are gold plated.

The transition from ridge gap waveguide to microstrip is achieved via the electromagnetic coupling established when overlapping a ridge section with a microstrip line segment. These transmission line segments are not in physical contact, i.e., constituting a contactless transition. Therefore, the coupling is achieved through the existent air gap between the 


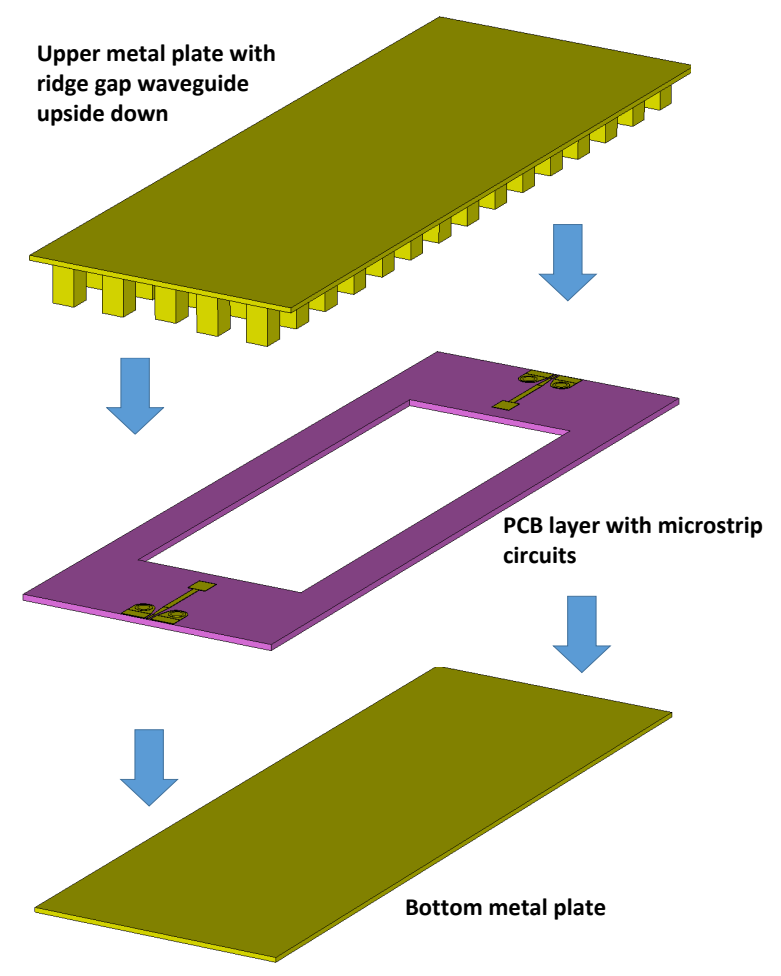

Fig. 2. Different layers of F-band transition.

ridge and the microstrip line. Since the gap between the bottom side of the pin surface and the lower metal plate is $0.2 \mathrm{~mm}$ (as represented in Fig. 1(a)), considering the thickness of the substrate and the thickness of the metal strips in the PCB $(10 \mu \mathrm{m})$, the separation between the ridge and the microstrip segment is then $\mathrm{g} 2=63 \mu \mathrm{m}$ as ilustrated in Fig. 3. The distance between the top side of the substrate and the bottom face of the pin is $\mathrm{g} 1=73 \mu \mathrm{m}$.

There are two circuits placed at both sides of the PCB (see Fig. 4) in order to analyze the transitions in back-toback configuration. Each of these circuits is composed of two sections. First, a transition between CPW and microstrip is needed since the measurements will be performed by using Ground-Signal-Ground (GSG) wafer probes. The dimensions of the coplanar waveguide have been chosen in such a way that an input impedance of $50 \Omega$ is ensured as well as the pitch in the CPW circuit is kept below $100 \mu \mathrm{m}$. The transition from CPW to microstrip is designed by means of an intermediate tapered section whose width is gradually increased to match the width of a $50 \Omega$ microstrip line allowing a smooth field transformation. We have included via holes that connect the ground pads of the CPW to the ground plane in order to suppress potential parallel plate modes. The second part of the transition is a $50 \Omega$ microstrip line terminated by a rectangular patch. This patch constitutes a matching section that overlaps a certain ridge segment, which is located in the upper layer. Therefore, the complete transition geometry includes a CPWto-microstrip interface and a microstrip-to-ridge gap waveguide

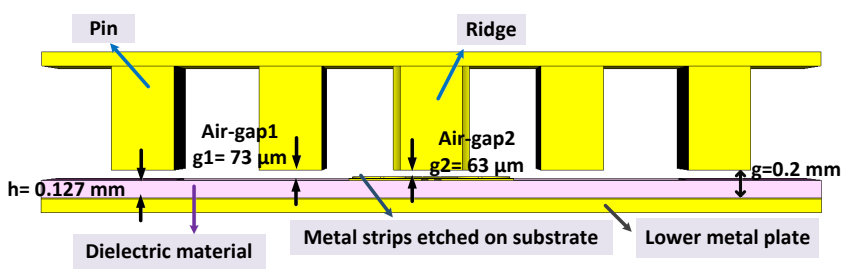

Fig. 3. Front view of complete transition geometry.

transition. Fig. 4 shows that a certain central area of the PCB has been cut out, allowing the electromagnetic fields to propagate along and between the ridge gap waveguide and the lower metal plate through air media. At the same time, we keep both circuits that constitute the back-to-back transitions in the same PCB instead of having two separate PCBs. This ensures that we get a better mechanical alignment between the two circuits, since different boards would need to be soldered separately and that involves a higher risk to get poor alignment. The dimensions of the CPW-microstrip circuits are summarized in Table I.

The ridge is composed of three sections: a central ridge with length equal to $11.6 \mathrm{~mm}$ (approximately $4 \lambda$ at $100 \mathrm{GHz}$ ), and two additional side ridge sections that overlap the microstrip patches of the PCB layer. One side ridge section receives the signal coupled from the microstrip patch, the main ridge guides the signal to the output, and then the second side ridge section couples the signal to the second microstrip patch. The length of the microstrip patch and the side ridge section have been initially set as $\lambda_{g} / 4$ and $\lambda / 4$ respectively (where $\lambda_{g}=1.12$ $\mathrm{mm}$ and $\lambda=3 \mathrm{~mm}$ for $100 \mathrm{GHz}$ ). Afterwards, a parametric sweep of that length, the width of the microstrip segments and the width of the side ridges is performed in order to improve the matching. We have observed that we get best transition performance when the width of the side ridges follows the shape of the microstrip patch. Fig. 5 illustrates the top view of the complete geometry, as well as the overlapped area between the microstrip section and the ridge gap waveguide. We have also placed two extra pins before and after the ridge with the aim to maintain the microstrip line properly packaged avoiding possible radiation.

\section{Simulation AND MEASUREMENT RESUlts}

The proposed transition geometry has been numerically analyzed in terms of S-parameters by using CST Microwave Studio. The simulation result of this structure is presented in Fig. 6. The simulation shows that return loss is larger than 15 $\mathrm{dB}$ in about $23.6 \%$ relative bandwidth for the gap waveguide prototype. The resulting insertion loss is lower than $1.3 \mathrm{~dB}$ over this bandwidth, which implies $0.65 \mathrm{~dB}$ for a single transition. Note that the contribution from the transition from CPW to microstrip is included but the actual objective is to design a transition from microstrip to ridge gap waveguide. Therefore, the insertion loss of our transition is lower if we exclude the loss of the CPW-microstrip section, which is possible by applying a Thru-Reflect-Line (TRL) de-embedding method. 


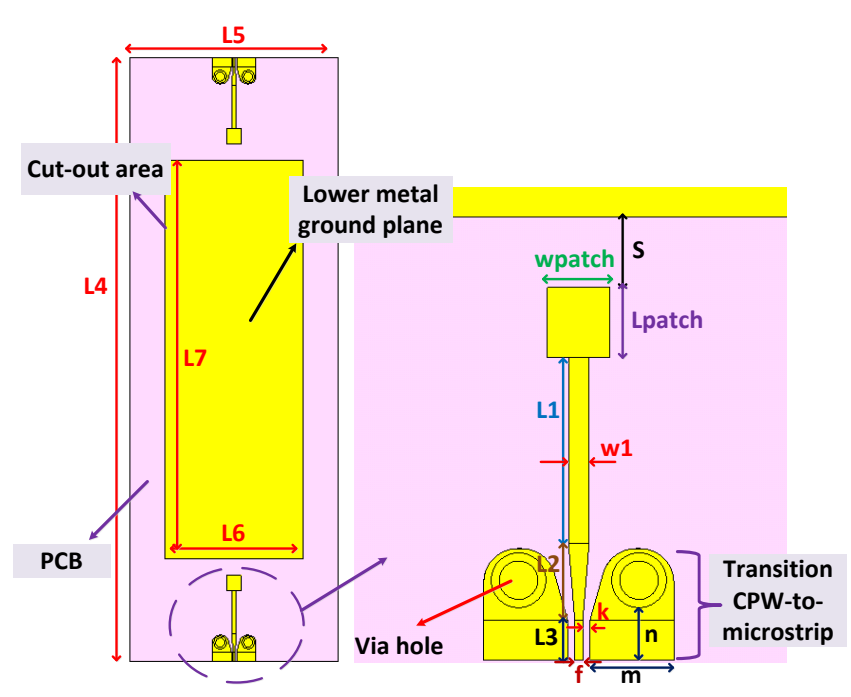

Fig. 4. Top view of PCB and design details of CPW-microstrip circuit.

TABLE I. DIMENSIONS OF PCB LAYER.

\begin{tabular}{|c|c||c|c|}
\hline Parameter & Value $(\mathrm{mm})$ & Parameter & Value $(\mathrm{mm})$ \\
\hline L1 & 1.16 & Lpatch & 0.39 \\
\hline L2 & 0.48 & wpatch & 0.44 \\
\hline L3 & 0.25 & w1 & 0.13 \\
\hline L4 & 16.29 & S & 0.44 \\
\hline L5 & 5.6 & $\mathrm{f}$ & 0.05 \\
\hline L6 & 3.7 & $\mathrm{k}$ & 0.05 \\
\hline L7 & 10.73 & $\mathrm{~m}$ & 0.52 \\
\hline & & $\mathrm{n}$ & 0.33 \\
\hline
\end{tabular}

The gap waveguide layers have been manufactured by using a Computer Numerical Control (CNC) milling machine. Milling is a very precise manufacturing technique and the tolerance in the pin/ridge size, height and period has been found to be in the order of $\pm 1 \mu \mathrm{m}$. The PCBs have been fabricated by standard photolithography process. According to the specifications from the PCB manufacturer (CoorsTek), the dimensions of the etched strips as well as the existent gaps between the ground pads and signal pad that constitute the $\mathrm{CPW}$, show a tolerance of $\pm 2.54 \mu \mathrm{m}$ (verified by using a microscope). The via holes of the CPW ground pads have been realized by laser drilling and are gold plated. The central area of the PCB has been cut-out by laser as well. The most critical tolerances regarding the PCB manufacturing have been found to be the corresponding ones to the via hole drilling (hole diameter and hole location have a tolerance of $\pm 51 \mu \mathrm{m}$ ), and the overall substrate dimensions $( \pm 25.4 \mu \mathrm{m})$. The substrate is actually $18 \mu \mathrm{m}$ thicker than the selected standard one, the metal strip is $6 \mu \mathrm{m}$ thinner and the ground plane is $5 \mu \mathrm{m}$ thicker. These tolerance values are quite significant compared to the overall circuit dimensions and the wavelength value at the frequency of operation. Therefore, tolerances will certainly have an impact on the circuit performance.

A picture of the manufactured prototype including soldered

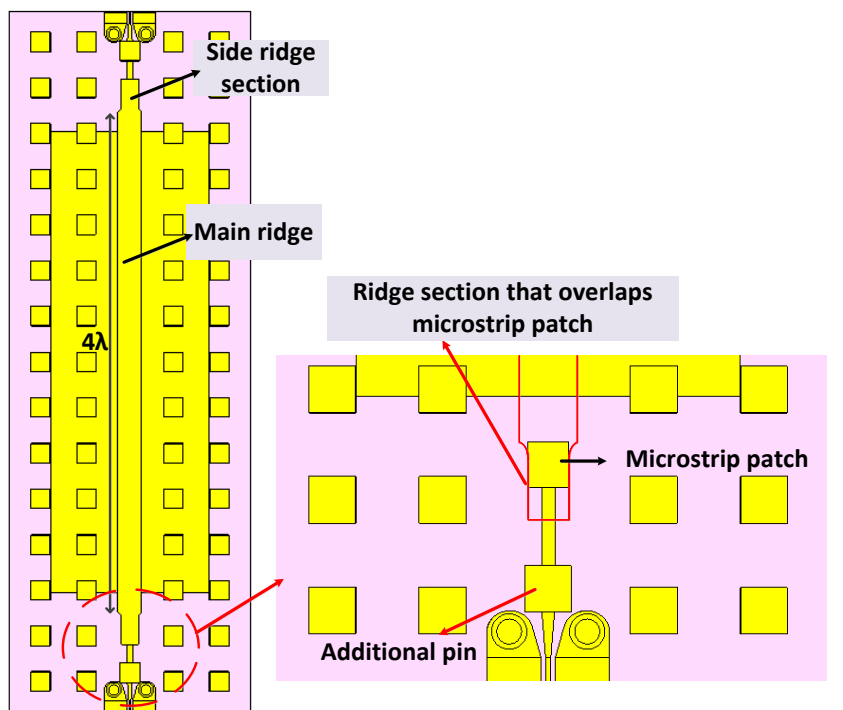

Fig. 5. Top view of transition prototype (top metal lid is hidden to allow visualization of design details).

PCB to the metal plate is shown in Fig. 7. The assembly of the metal blocks is done by using four screws. The PCB needs to be suitably attached to a metal plate as illustrated in Fig. 8. The PCB is placed between two side steps of thickness equal to $0.5 \mathrm{~mm}$ that, together with the side steps of the upper metal plate (of thickness equal to $0.47 \mathrm{~mm}$ ), contribute to keep the required gap constant. During this assembly process, it is found difficult to keep the air-gap distance constant because the soldering liquid might add up to several tens of micrometers of thickness below the PCB, thus lifting the board in an uneven way which results in a smaller air-gap at some points of the prototype. A decrease in the air-gap constitutes a change in the transition performance since the coupling between the microstrip patch and the ridge section becomes different. One solution to counteract this effect is to increase the depth of the groove of the bottom metal plate that exists between the side steps (see Fig. 8) where the PCB is soldered, and estimate in advance how much thickness the soldering liquid might add. However, there is an unavoidable human factor on the soldering process and it is difficult to achieve exactly $73 \mu \mathrm{m}$ of constant gap. After soldering, the straight ridge gap waveguide prototype shows a gap of $50 \mu \mathrm{m}$ at both sides.

The fabricated F-band prototypes have been measured by employing wafer-probe stations equipped with coplanar launchers and a Vector Network Analyzer (VNA) together with two frequency extension modules as shown in Fig. 9. A comparison between simulated and experimental transmission properties is plotted in Fig. 10. In order to achieve a fairer comparison, we have introduced the known data regarding tolerances (via hole diameter, substrate and metal strips characteristics, ground plane thickness, and obtained airgaps after soldering) in our CST model. The first observation on the comparison between simulations and measurements is a degradation on the prototype performance with respect to the simulation results presented in Fig. 6, clearly caused by the 
PCB manufacturing and assembly tolerances.

In the obtained experimental results shown in Fig. 10(a) we can identify a resonance peak at $93 \mathrm{GHz}$ that has been observed in all measurements. One reason that could explain the origin
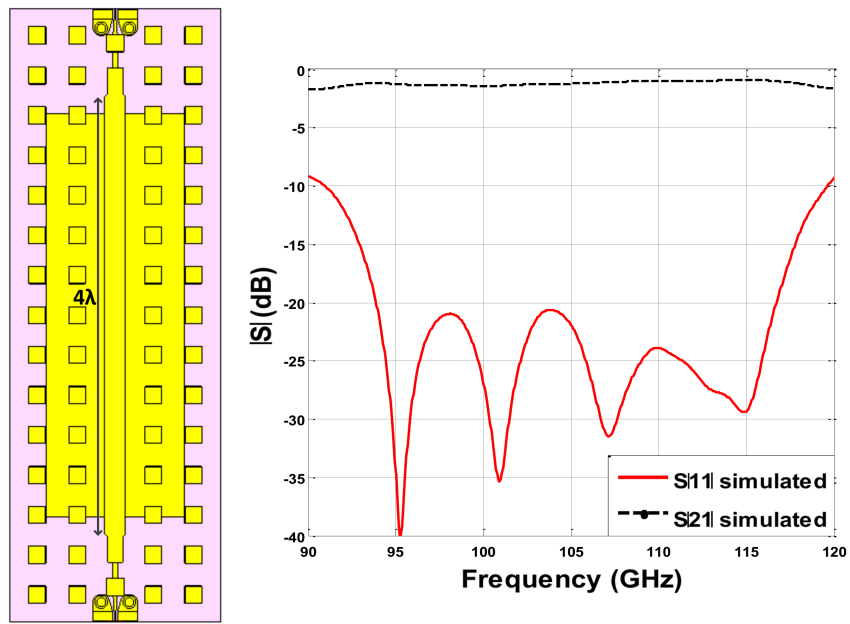

Fig. 6. Simulated S-parameters of transition geometry with straight ridge gap waveguide.

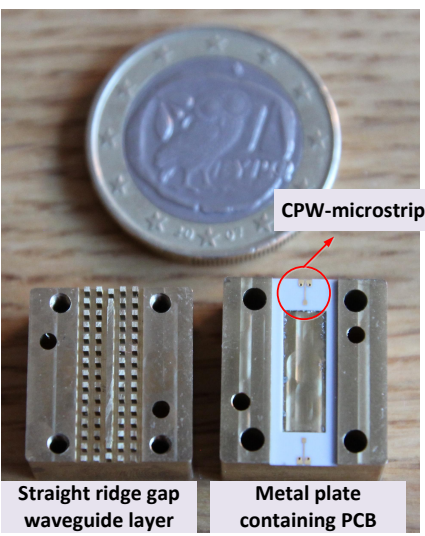

Fig. 7. Straight ridge gap waveguide and corresponding PCB soldered into metal layer.

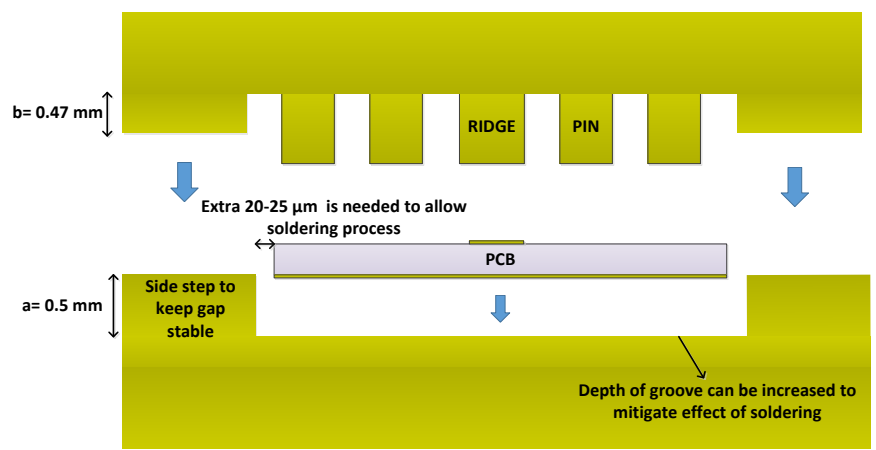

Fig. 8. Description of assembly of mechanical parts for the straight ridge gap waveguide prototype. of the resonance is that the ground pads of the CPW behave as two patch antennas with a coplanar feed along one side of each patch. This resonance phenomena was studied in [20] and [21] where an expression to obtain the resonance frequencies is given. The resonance frequency depends on the width and length of the CPW ground pad. Using that expression and our corresponding pad dimensions we obtain the first resonance at $91.8 \mathrm{GHz}$, which is very close to the measured resonance.

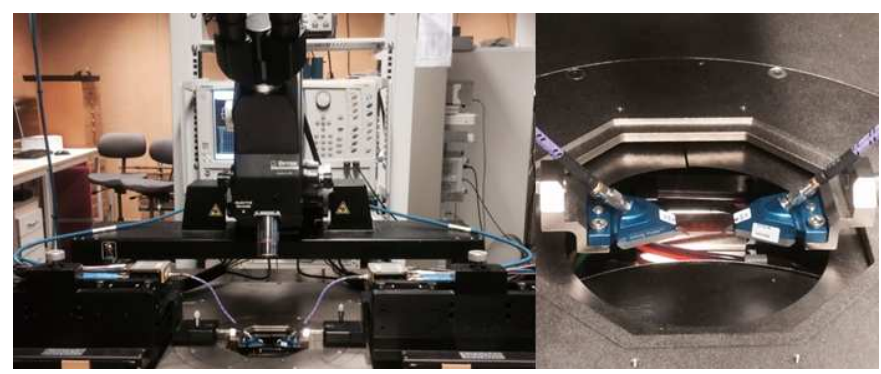

Fig. 9. Measurement setup for F-band prototype on wafer-probe station. Full setup view is shown to the left and the probes are shown to the right.

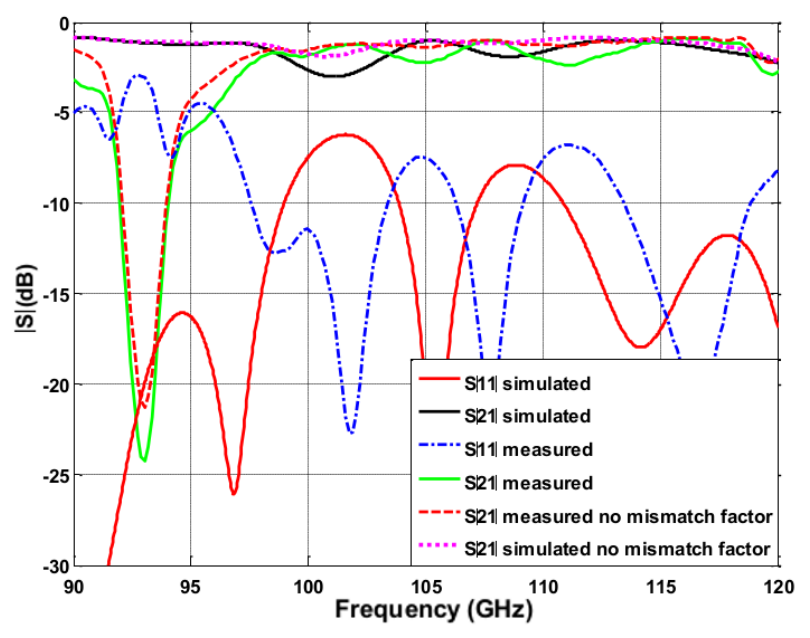

(a)

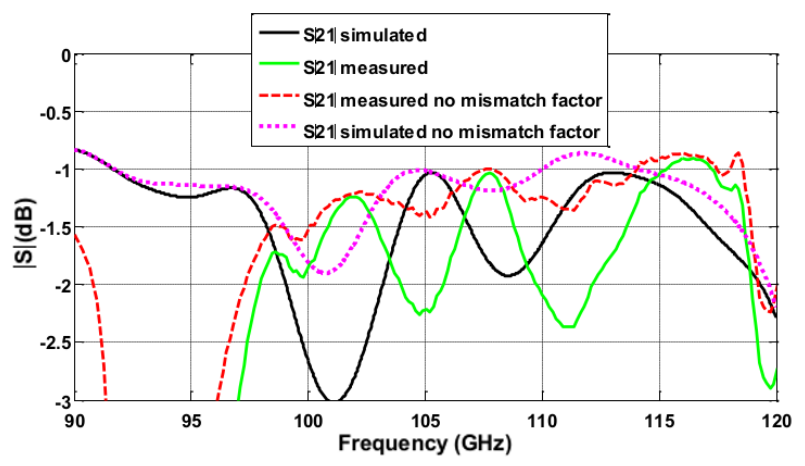

(b)

Fig. 10. (a) Simulated and measured S-parameters of back-to-back transition between microstrip and straight ridge gap waveguide. (b) Zoomed Sparameters to visualize insertion loss. 
Thereby, it could be possible that the ground CPW pads radiate energy causing the resonance. In spite of this resonance peak and a small frequency shift of about $3 \mathrm{GHz}$, simulated and measured return loss are about same level between $98 \mathrm{GHz}$ and $119 \mathrm{GHz}$. Fig. 10(b) represents a zoomed view of the simulated and measured $\left|S_{21}\right|$ parameter. By extracting the mismatch factor to the $\left|S_{21}\right|$ values we obtain the dissipative contribution of the loss, which is smaller than $1.9 \mathrm{~dB}$ for both simulations and measurements in the frequency interval 98 $119 \mathrm{GHz}$

\section{Monte CARlo TOlerance AnAlysis}

Measurement results show a degradation of the transition performance most likely due to PCB manufacturing and prototype assembly tolerances. For mechanical design purpose and manufacturing specifications, it is exemplified in related areas [22] - [24], that Monte Carlo based tolerance analysis is a useful approach in multi-parameter analysis [25]. Our measurement results indicate that misalignments in the air gap between the ridge and the PCB during the assembly of the prototype is of distinct importance. Furthermore, the horizontal displacement of the PCB with respect to the ridge gap waveguide caused during the soldering process could also be a critical factor that affects the transition performance. Our aim is to identify the most critical parameters in order to give suitable guidelines on designing the mechanical parts of gap waveguides at mm-wave frequencies. A MATLAB/CST code is created to emulate these misalignment factors on our transition design over a large set of samples. The MATLAB script modifies the CST model tolerance parameters for each sample and logs the output results for statistical analysis. We implement the Monte Carlo technique to draw random values from specified distributions for all displacement parameters under test independently of each other. Uniform distribution is considered to efficiently evaluate a worst case scenario and identify the most critical tolerance parameter. CST Microwave Studio is a Finite Difference Time Domain (FDTD) electromagnetic solver, and therefore, the mesh resolution is important to consider in order to have a valid tolerance analysis. The random misalignment change must be larger than the smallest mesh step in the model. This will be detailed in section IV-B.

\section{A. Tolerance parameters under test}

Three types of assembly misalignments are included in this analysis: a) the gap misalignment between the top PCB surface and the bottom of the pins at each port with respect to the nominal air gap of $73 \mu \mathrm{m}$ (Fig. 11(a)); b) PCB horizontal translational misalignment (Fig. 11(b)); c) PCB rotational misalignment around the center point of the horizontal face (Fig. 11(c)). We describe the gap waveguide performance as a function $W$ of these five tolerance parameters:

$$
\left|S_{x x}\right|=W\left(\Delta_{g 1}, \Delta_{g 2}, \Delta_{t x}, \Delta_{t z}, \Delta_{r}\right)
$$

where $\Delta_{g 1}$ and $\Delta_{g 2}$ are random gap misalignments at ports 1 and 2 respectively, $\Delta_{t x}$ and $\Delta_{t z}$ are the PCB translational

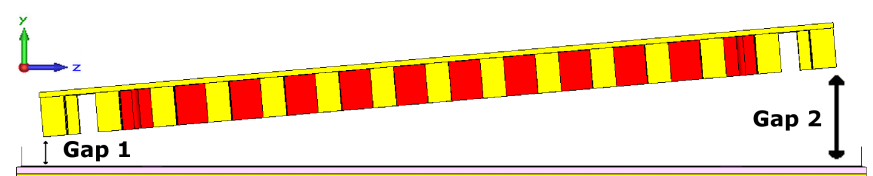

(a)

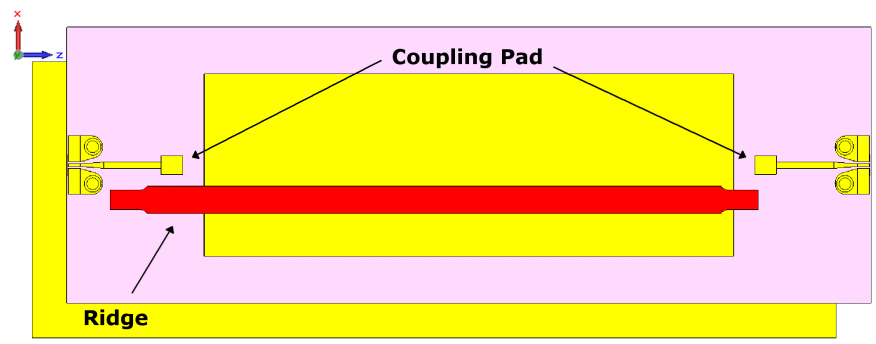

(b)

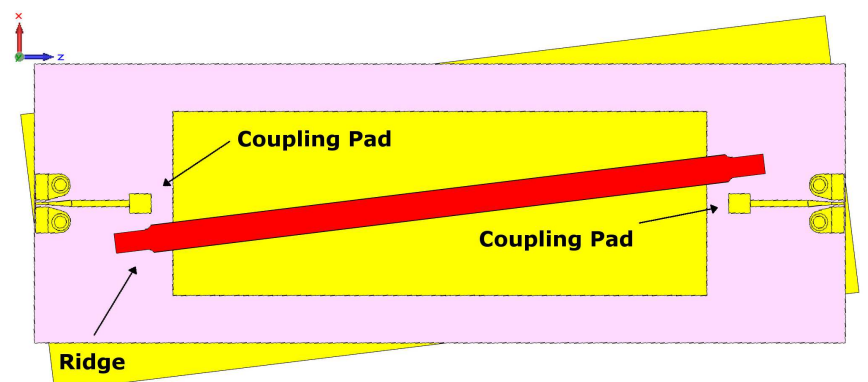

(c)

Fig. 11. Misalignment illustrations for tolerance parameter definitions. (a) Side view of gap misalignment $\Delta_{g 1}$ and $\Delta_{g 2}$. (b) Top view of PCB horizontal translational misalignments $\Delta_{t x}$ and $\Delta_{t z}$. Top lid and pins are hidden for allowing ridge and PCB visualization. (c) Top view of PCB rotational misalignment $\Delta_{r}$. Top lid and pins are hidden for allowing ridge and PCB visualization. Misalignment is exaggerated for illustration purpose.

misalignments along $\mathrm{X}$ and $\mathrm{Z}$ directions, respectively, and $\Delta_{r}$ the angle of the PCB rotational misalignment. $\left|S_{x x}\right|$ represents the reflection and transmission coefficient output.

\section{B. Mesh and tolerance resolution}

To be confident that the misalignment under test is meshed properly, the smallest tolerance step (smallest misalignment) in the simulation, $\Delta_{s t s}$, must be larger than the smallest mesh step, $\Delta_{s m s}$, in the mesh grid. The importance of this is illustrated in Fig. 12. To ensure that simulated tolerances in this study were valid, a large set of pre-samples were created. From these we evaluated that $\Delta_{s m s}=2.3 \mu \mathrm{m}$ or smaller for all the samples. This means that with $\Delta_{s t s}=3 \mu \mathrm{m}$ that was used, we are confident that the simulations are valid.

\section{Tolerance intervals under test}

The tolerance intervals under test have been extracted from our own observations when assembling the manufactured prototypes. The most extreme gap misalignment $\left(\Delta_{g 1}, \Delta_{g 2}\right)$ was measured to be in the interval $[-23,47] \mu \mathrm{m}$ from nominal 


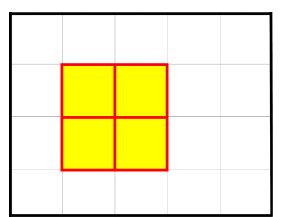

(a)

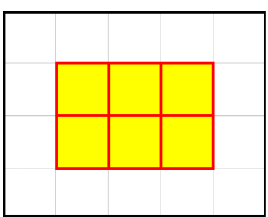

(b)

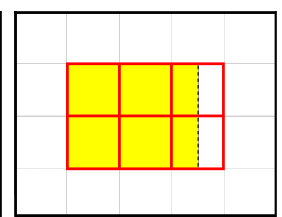

(c)
Fig. 12. (a) Mesh resolution is good and the object (yellow rectangle) is completely covered. (b) Object is extended with an extra column. The grid covers the entire object again and simulation would still be valid. (c) Object is extended but not enough and falls between grid lines, this particular simulation cannot be guaranteed to give accurate results.

gap distance of $73 \mu \mathrm{m}$. The PCB is soldered within a groove that is $\pm 20 \mu \mathrm{m}$ wider than the PCB width, therefore PCB translational misalignment $\left(\Delta_{t x}, \Delta_{t z}\right)$ in the interval $[-20,20]$ $\mu \mathrm{m}$ and rotational $\left(\Delta_{r}\right)$ within $\left[-0.5^{\circ}, 0.5^{\circ}\right]$ from the nominal design were considered for investigation. The smallest tolerance step was, as mentioned previously, set to $\Delta_{\text {sts }}=3$ $\mu \mathrm{m}$ for the gap and translational PCB tolerance parameters. The step of the rotational misalignment is set as $0.01^{\circ}$ by considering the misaligned size at the ridge ends due to the linear scaling along the length of the waveguide.

\section{TOLERANCE ANALYSIS RESULTS}

The S-parameters obtained from 719 samples, which constitutes the complete simulation set, is represented with upper and lower (nominal) limit in Fig. 13. The corresponding tolerance parameter distributions in histogram form are shown in Fig. 14. Table II represents a summary of the tolerance

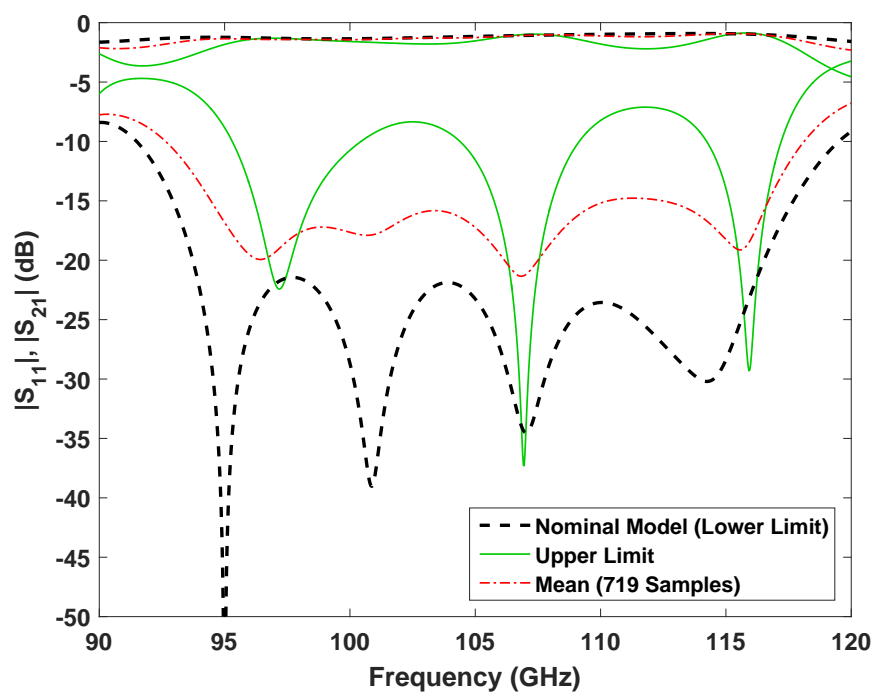

Fig. 13. 719 simulation samples (full sample set) represented with upper and lower (nominal) limit of S-Parameters. Black dotted line represents the nominal performance.

intervals for each parameter under study and the mean, Standard Deviation (STD) and Root Mean Square (RMS) of the parameter distributions extracted from the obtained sample data shown in Fig. 13.

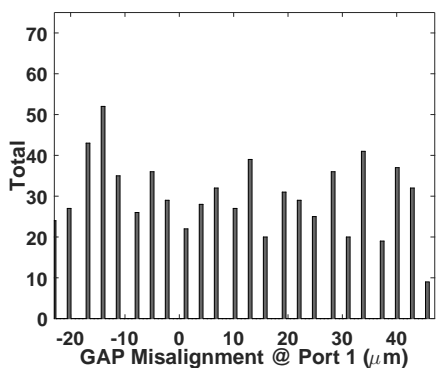

(a) $\Delta_{g 1}$

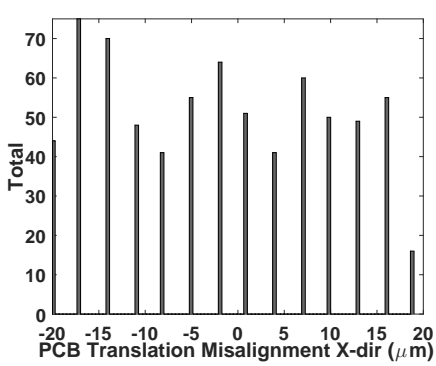

(c) $\Delta_{t x}$

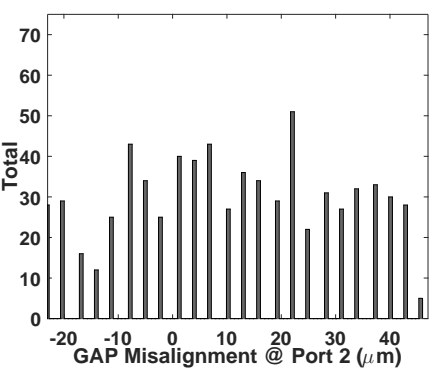

(b) $\Delta_{g 2}$

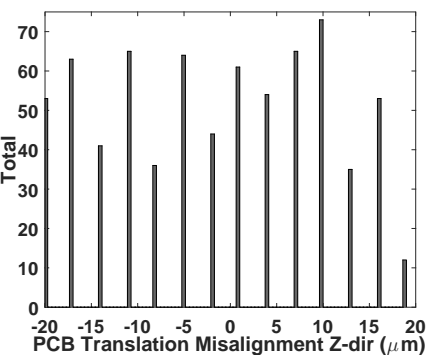

(d) $\Delta_{t z}$

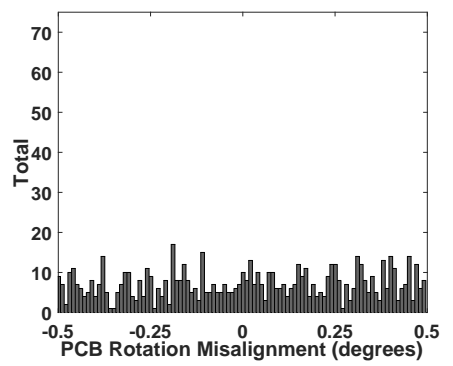

(e) $\Delta_{r}$

Fig. 14. Histogram distribution of the five tolerance parameters, for the 719 simulation samples of Fig. 13. Parameters are displayed as misalignment from nominal value.

TABLE II. DISTRIBUTION STATISTICS OF THE FIVE TOLERANCE PARAMETERS FOR THE 719 SIMULATION SAMPLES OF FIG. 13 PARAMETERS ARE DISPLAYED AS MISALIGNMENT FROM NOMINAL VALUE.

\begin{tabular}{|l|c|c|c|c|c|}
\hline & Min & Max & Mean & STD & RMS \\
\hline$\Delta_{g 1}(\mu \mathbf{m})$ & -23.00 & 46.00 & 9.63 & 20.51 & 22.64 \\
\hline$\Delta_{g 2}(\mu \mathbf{m})$ & -23.00 & 46.00 & 11.33 & 19.02 & 22.13 \\
\hline$\Delta_{t x}(\mu \mathbf{m})$ & -20.00 & 19.00 & -2.00 & 11.62 & 11.78 \\
\hline$\Delta_{t z}(\mu \mathbf{m})$ & -20.00 & 19.00 & -1.56 & 11.37 & 11.47 \\
\hline$\Delta_{r}\left(^{\circ}\right)$ & -0.50 & 0.49 & 0.01 & 0.29 & 0.29 \\
\hline
\end{tabular}

If we set the requirement for $\left|S_{11}\right|<-15 \mathrm{~dB}$ over $95-115$ GHz, 242 samples out of the total 719 fulfill it. Fig. 15 and 16 show the reflection and transmission coefficients represented by upper and lower (nominal) limit and the tolerance parameter distribution of the 242 cases that fulfilled the previous condition, respectively. We can observe in Fig. 16 that $\Delta_{t x}$, $\Delta_{t z}$ and $\Delta_{r}$ are approximately uniform distributed over the 


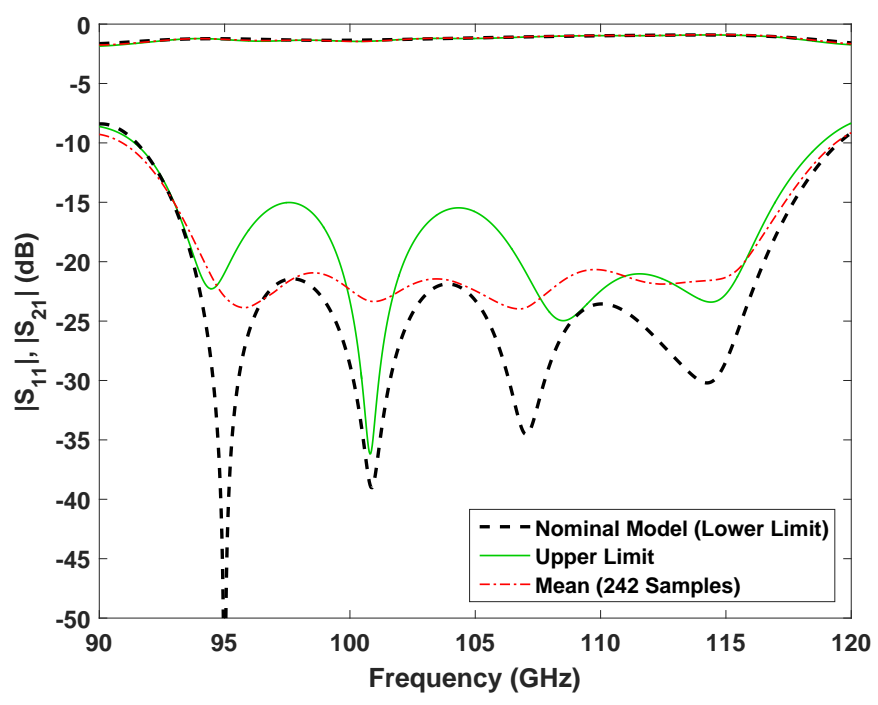

Fig. 15. 242 simulation samples out of $719(\approx 33 \%)$ show $\left|S_{11}\right|<-15$ $\mathrm{dB}$ from 95 to $115 \mathrm{GHz}$, here represented with upper and lower (nominal) limit of S-Parameters. Black dotted line represents the nominal performance.

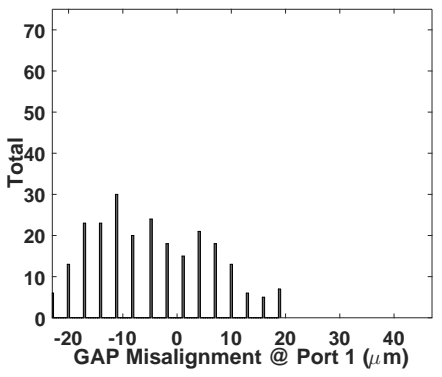

(a) $\Delta_{g 1}$

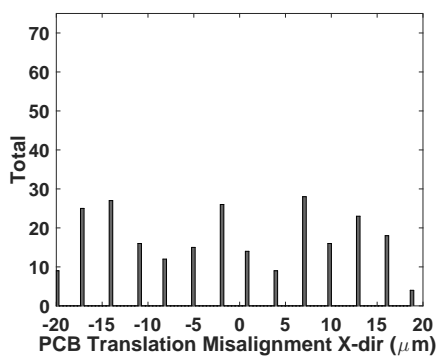

(c) $\Delta_{t x}$

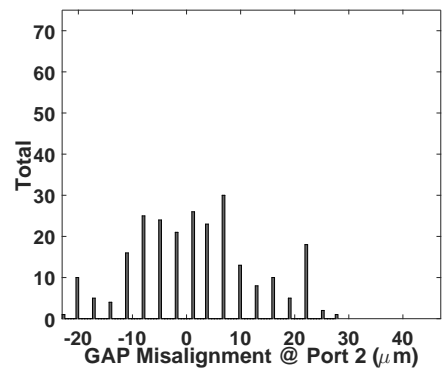

(b) $\Delta_{g 2}$

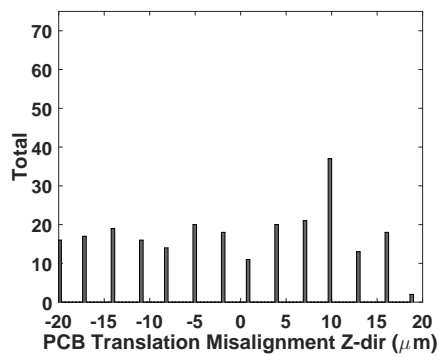

(d) $\Delta_{t z}$

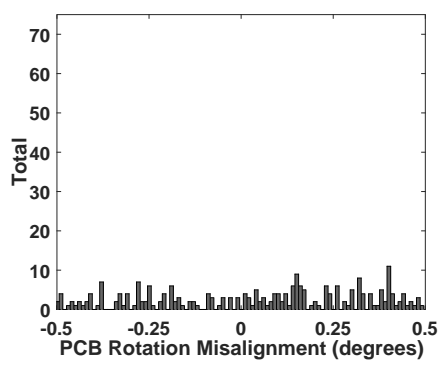

(e) $\Delta_{r}$

Fig. 16. Histogram distribution of the five tolerance parameters, for the 242 simulation samples of Fig. 15. Parameters are displayed as misalignment from nominal value.
TABLE III. DISTRIBUTION STATISTICS OF THE FIVE TOLERANCE PARAMETER HISTOGRAMS FOR THE 242 SIMULATION SAMPLES OF FIG. 15. PARAMETERS ARE DISPLAYED AS MISALIGNMENT FROM NOMINAL VALUE.

\begin{tabular}{|l|c|c|c|c|c|}
\hline & Min & Max & Mean & STD & RMS \\
\hline$\Delta_{g 1}(\mu \mathbf{m})$ & -23.00 & 19.00 & -4.59 & 10.66 & 11.58 \\
\hline$\Delta_{g 2}(\mu \mathbf{m})$ & -23.00 & 28.00 & 1.61 & 11.15 & 11.25 \\
\hline$\Delta_{t x}(\mu \mathbf{m})$ & -20.00 & 19.00 & -1.29 & 11.52 & 11.57 \\
\hline$\Delta_{t z}(\mu \mathbf{m})$ & -20.00 & 19.00 & -0.81 & 11.32 & 11.32 \\
\hline$\Delta_{r}\left(^{\circ}\right)$ & -0.50 & 0.49 & 0.04 & 0.28 & 0.29 \\
\hline
\end{tabular}

tolerance interval under test (section IV-C), whilst $\Delta_{g 1}$ and $\Delta_{g 2}$ show a Gaussian distribution over $[-23,28] \mu \mathrm{m}$, with a STD of $11 \mu \mathrm{m}$ approximately (see Table III). This means that $\Delta_{g 1}$ and $\Delta_{g 2}$ are the two most critical tolerance parameters for the assembly tolerance. We have also identified that the tolerance interval $[-23,28] \mu \mathrm{m}$ for $\Delta_{q 1}$ and $\Delta_{q 2}$ does not guarantee a reflection coefficient below $-15 \mathrm{~dB}$; there are 445 samples with $\Delta_{g 1}$ and $\Delta_{g 2}$ within $[-23,28] \mu \mathrm{m}$. This means that approximately $54 \%$ of the samples with $\Delta_{g 1}, \Delta_{g 2}$ within $[-23,28] \mu \mathrm{m}$ (together with some random combination of the other three misalignments $\Delta_{t x}, \Delta_{t z}$ and $\Delta_{r}$ ) achieve the requirement of $\left|S_{11}\right|<-15 \mathrm{~dB}$. Therefore, we have extracted several cumulative distribution function (CDF) curves for different $\Delta_{g 1}$ and $\Delta_{g 2}$ tolerance intervals, as shown in Fig. 17. If we set the tolerance interval of $\Delta_{g 1}, \Delta_{g 2}$ to $[-10,10]$ $\mu \mathrm{m}$ together with the other misalignment tolerances (as defined in section IV-C), the possibility to fulfill the requirement of $\left|S_{11}\right|<-15 \mathrm{~dB}$ is $98 \%$, close to $100 \%$.

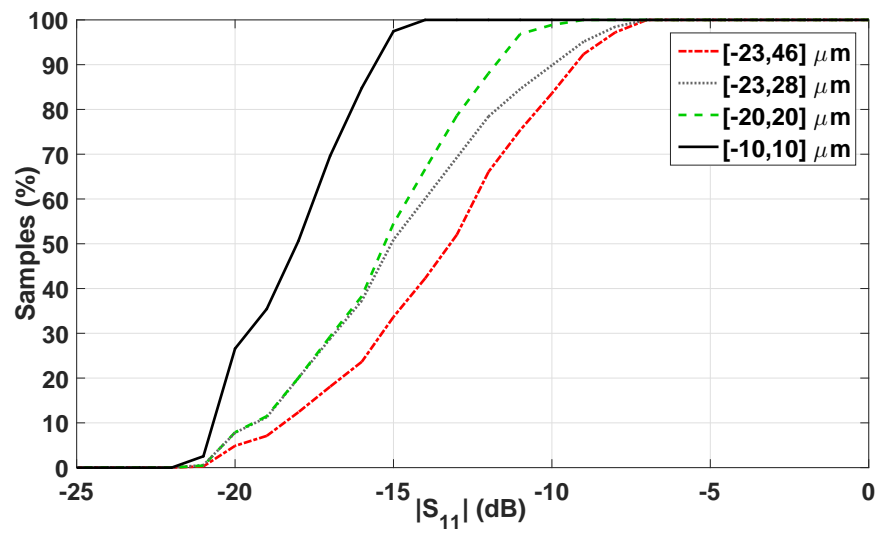

Fig. 17. Each CDF curve represents obtained samples when both gaps misalignment have values within the interval specified in the legend. The curves show the percentage of these samples (within each interval) where $\left|S_{11}\right|$ performance is better than a certain $\mathrm{dB}$ level (x-axis) for the frequency band of $95-115 \mathrm{GHz}$. The red dash-dotted curve represents all the samples and hence the largest misalignment interval.

\section{CONCLUSION}

We have proposed an F-band transition from standard microstrip to ridge gap waveguide via electromagnetic coupling. The transition has been numerically analyzed in back-to-back structure, showing return loss larger than $15 \mathrm{~dB}$ over more than $23 \%$ relative bandwidth, and insertion loss lower than 
$1.3 \mathrm{~dB}$. Assembly and PCB manufacturing tolerances degrade the transition performance as seen in the presented comparison between simulations and measurements. Still, we could achieve a reasonably good agreement between simulated/measured return loss and dissipation factor in about $15 \%$ relative bandwidth.

The presented Monte Carlo tolerance analysis establishes guidelines and requirements for the assembly process. It is clear from this study that the misalignment in the gap distance is the most important tolerance factor that affects the S-parameter performance. We can conclude that a tolerance interval for gap misalignment within $[-10,10] \mu \mathrm{m}$ (which implies $[-\lambda / 300, \lambda / 300]$ in terms of wavelength), PCB translational misalignment in both horizontal directions within $[-20,20] \mu \mathrm{m}([-\lambda / 150, \lambda / 150])$ and PCB rotational misalignment within $\left[-0.5^{\circ}, 0.5^{\circ}\right]$ during assembly gives a possibility close to $100 \%$ to fulfill the performance requirement $\left|S_{11}\right|<-15 \mathrm{~dB}$ over the frequency band of $95-115$ GHz. Another relevant conclusion from this work is that this transition prototype is assembled in such a way that gap misalignments are very probable to happen. Therefore, the assembly process should be considered in advance when designing high frequency gap waveguide prototypes in order to mitigate the misalignments as much as possible by paying special attention to how to stabilize the air-gap.

In spite of the observed issues related to the assembly tolerances, this proposed transition becomes an interesting solution for integrating MMICs with ridge gap waveguide circuits. The gap waveguide interface keeps the active components suitably packaged and provides electrical isolation among adjacent RF chips avoiding any possible interference or coupling. The next step of this work will constitute the investigation of the employment of the presented transition to interconnect an amplifier to a ridge gap waveguide.

\section{ACKNOWLEDGMENT}

The authors would like to thank the precision mechanician Carl-Magnus Kihlman from the Microtechnology and Nanoscience (MC2) department at Chalmers University for fabricating the gap waveguide blocks, as well as helping us during the assembly of the gap waveguide prototypes. We also want to thank Prof. Enoksson and Lic. Eng. Rahiminejad from MC2 dep., Dr. Uz Zaman from Signals and Systems dep. and Dr. Haasl for their useful feedback during the project meetings.

\section{REFERENCES}

[1] K. J. Herrick, Y. Jong-Gwan, and L. P. B. Katehi, "Microtechnology in the development of three-dimensional circuits," IEEE Transactions on Microwave Theory and Techniques, Vol. 46, No. 11, pp. 1832 - 1844, 1998.

[2] A. V. Raisanen, D. Choudhury, R. J. Dengler, J. E. Oswald, and P. H. Siegel, "A novel split-waveguide mount design for millimeter- and submillimeter-wave frequency multipliers and harmonic mixers," IEEE Microwave and Guided Wave Letters, Vol. 3, No. 10, pp. 369 - 371, 1993.

[3] P.-S. Kildal, E. Alfonso, A. Valero-Nogueira, and E. Rajo-Iglesias, "Local metamaterial-based waveguides in gaps between parallel metal plates," IEEE Antennas and Wireless Propagation Letters, Vol. 8, No. 1, pp. 84 - 87, 2009.
[4] P.-S. Kildal, "Three metamaterial-based gap waveguides between parallel metal plates for $\mathrm{mm} / \mathrm{submm}$ waves," $3^{\text {rd }}$ European Conference on Antennas and Propagation EUCAP 2009, Berlin, Germany, 23 - 27 March 2009.

[5] E. Rajo-Iglesias and P.-S. Kildal, "Numerical studies of bandwidth of parallel plate cut-off realized by bed of nails, corrugations and mushroom-type EBG for use in gap waveguides," IET Microwaves, Antennas \& Propagation, Vol. 5, No. 3, pp. 282 - 289, March 2011.

[6] E. Pucci, A. Uz Zaman, E. Rajo-Iglesias, P.-S. Kildal, and A. Kishk, "Study of Q-Factors of Ridge and Groove Gap Waveguide Resonators," IET Microwaves, Antennas \& Propagation, Vol. 7, No. 11, pp. 900 908, 2013.

[7] E. Rajo-Iglesias, A. Uz Zaman, and P.-S. Kildal, "Parallel plate cavity mode suppression in microstrip circuit packages using a lid of nails," IEEE Microwave and wireless Components Letters, Vol. 20, No. 1, pp. 31 - 33, December 2010.

[8] A. Algaba Brazález, A. Uz Zaman, and P.-S. Kildal, "Improved microstrip filters using PMC packaging by lid of nails," IEEE Transactions on Components, Packaging and Manufacturing Technology, Vol. 2, No. 7, pp. 1075 - 1084, 2012.

[9] A. U. Zaman, T. Vukusic, M. Alexanderson, and P.-S. Kildal, "Gap Waveguide PMC packaging for improved isolation of circuit components in high frequency microwave modules," IEEE Transactions on Components, Packaging and Manufacturing Technology, Vol. 1, No. 9, pp. 16 - 25, 2012.

[10] A. U. Zaman, E. Rajo-Iglesias, E. Alfonso, and P.-S. Kildal, "Design of transition from coaxial line to ridge gap waveguide," IEEE Antennas and Propagation Society International Symposium, APSURSI, pp. 1 - 4, 2009.

[11] P.-S. Kildal, A. Uz Zaman, E. Rajo-Iglesias, E. Alfonso, and A. ValeroNogueira, "Design and experimental verification of ridge gap waveguides in bed of nails for parallel plate mode suppression," IET Microwaves, Antennas \& Propagation, Vol. 5, No. 3, pp. 262 - 270, March 2011.

[12] H. Raza, J. Yang, P.-S. Kildal, and E. Alfonso, "Microstrip-ridge gap waveguide - study of losses, bends and transition to WR-15," IEEE Transactions on Microwave Theory and Techniques, Vol. 62, No. 9, pp. 1943 - 1952, 2014

[13] A. Algaba Brazález, E. Rajo-Iglesias, J.-L. Vazquez-Roy, A. Vosoogh, and P.-S. Kildal, "Design and Validation of Microstrip Gap Waveguides and Their Transitions to Rectangular Waveguide, for Millimeter-Wave Applications," IEEE Transactions on Microwave Theory and Techniques, Vol. 63, No. 12, pp. 4035 - 4050, 2015.

[14] A. Algaba Brazález, A. U. Zaman, and P.-S. Kildal, "Design of a coplanar waveguide-to-ridge gap waveguide transition via capacitive coupling," $6^{\text {th }}$ European Conference on Antennas and Propagation EUCAP 2012, Prague, Czech Republic, 26 - 30 March 2012.

[15] A. Algaba Brazález, A. U. Zaman, and P.-S. Kildal, "Investigation of a Microstrip-to-Ridge Gap Waveguide transition by electromagnetic coupling," IEEE Antennas and Propagation Society International Symposium, APSURSI, pp. 1 - 2, 2012.

[16] A. U. Zaman, T. Vukusic, M. Alexanderson, and P.-S. Kildal, "Design of a simple transition from microstrip to ridge gap waveguide suited for MMIC and antenna integration," IEEE Antennas and Wireless Propagation Letters, Vol. 12, pp. 1558 - 1561, 2013.

[17] R. W. Jackson and D. W. Matolak, "Surface-to-Surface Transition via Electromagnetic Coupling of Coplanar Waveguides," IEEE Transactions on Microwave Theory and Techniques, Vol. 35, No. 11, pp. 1027 - 1032, May 1987.

[18] G. Strauss and W. S. Menzel, "Millimeter-wave monolithic integrated circuit interconnects using electromagnetic field coupling," IEEE Transactions on Components, Packaging, and Manufacturing Technology, Vol. 19, No. 2, pp. 278 - 282, May 1996.

[19] T. J. Ellis, J. P. Raskin, L. P. B. Katehi, and G. M. Rebeiz, "A wideband CPW-to-microstrip transition for millimeter-wave packaging," 1999 IEEE MTT-S International Microwave Symposium Digest, Vol. 2, pp. 629 - 632, 1999. 
[20] W. H. Haydl, "Resonance phenomena and power loss in conductorbacked coplanar structures," IEEE Microwave and Guided Wave Letters, Vol. 10, No. 12, pp. 514 - 516, March 2000.

[21] W. H. Haydl, "On the use of vias in conductor-backed coplanar circuits," Transactions on Microwave Theory and Techniques, Vol. 50, No. 6, pp. 1571 - 1577, June 2002.

[22] J. Bornemann, U. Rosenberg, S. Amari, and R. Vahldieck, "Tolerance analysis of bypass-, cross- and direct coupled rectangular waveguide band-pass filters," IEE Proceedings Microwaves, Antennas and Propagation, Vol. 152, No. 3, pp. 167 - 170, June. 2005.

[23] A. Gonzalez and Y. Uzawa, "Tolerance analysis of ALMA band 10 corrugated horns and optics," IEEE Transactions on Antennas and Propagation, Vol. 60, No. 7, pp. 3137 - 3145, July 2012.

[24] M. Candotti, Y. Uzawa, S. V. Shitov, Y. Fujii, and K. Kaneko, "ALMA band 10 optics tolerance analysis," in Proc. 19th Int. Symp. on Space THz Technol., Groningen, The Netherlands, Apr. 2008, pp. 521 - 527.

[25] A. Hammar, M. Whale, P. Forsberg, A. Murk, A. Emrich, and J. Stake, "Optical Tolerance Analysis of the Multi-Beam Limb Viewing Instrument STEAMR," IEEE Transactions on Terahertz Science and Technology, Vol. 4, No. 6, pp. 714 - 721, 2014.

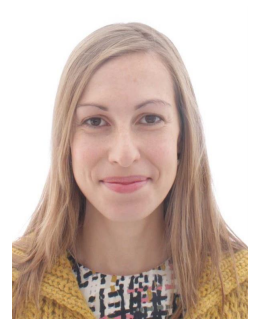

Astrid Algaba Brazález was born in Alicante, Spain, in 1983. She received her Telecommunication Engineering degree from Miguel Hernández University of Elche, Alicante, Spain, in 2009, and the Licentiate of Engineering and $\mathrm{PhD}$ degrees from Chalmers University of Technology, Gothenburg, Sweden, in 2013 and 2015, respectively. She has joined Ericsson Research, Ericsson AB, Gothenburg, Sweden, in November 2014. Her main research interests include the development of gap waveguide technology for millimeter and sub-millimeter wave applications, microwave passive gap waveguide components, packaging of microstrip filters, design of high frequency transitions between gap waveguide and other technologies, and metamaterials.

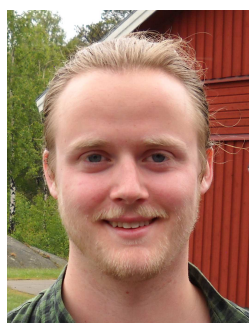

Jonas Flygare was born in Molndal, Sweden in 1988. He started his engineering physics studies for a B.Sc. and M.Sc. at Chalmers University of Technology, Gothenburg, Sweden in 2007 and 2010 respectively. In 2012, he was employed during a year as a test technician at CBRITE Inc. in Goleta, CA, USA where he was developing Thin-Film-Transistor technology for next generation flat screens. In 2014 he was employed at the antenna group, Signals and Systems department at Chalmers working with Ridge Gap Waveguide Tolerances. Since October 2014 he is employed as a Project Assistant at the Onsala Space Observatory, Onsala, Sweden operated by Chalmers. He is mainly involved in antenna design, simulation and optimization for the Square Kilometre Array project (SKA).

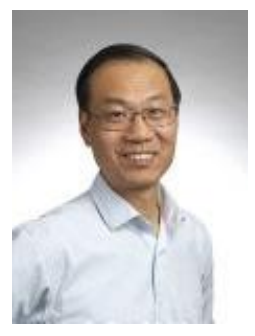

Jian Yang received the B.Sc. degree from the Nanjing University of Science and Technology, Nanjing, China, in 1982, and the M.Sc. degree from the Nanjing Research Center of Electronic Engineering, Nanjing, China, in 1985, both in electrical engineering, and the Swedish Licentiate and $\mathrm{PhD}$ degrees from the Chalmers University of Technology, Gothenberg, Sweden, in 1998 and 2001, respectively. From 1985 to 1996, he was with the Nanjing Research Institute of Electronics Technology, Nanjing, China, as a Senior Engineer. From 1999 to 2005, he was with the Department of Electromagnetics, Chalmers University of Technology as a Research Engineer. During 2005 and 2006, he was with COMHAT AB as a Senior Engineer. From 2006 to 2010, he was an Assistant Professor, and since 2010, he has been Associate Professor at the Department of Signals and Systems, Chalmers University of Technology. His research interests include $60-120 \mathrm{GHz}$ antennas, THz antennas, MIMO antennas, ultrawideband (UWB) antennas and UWB feeds for reflector antennas, UWB radar systems, UWB antennas in near-field sensing applications, hat-fed antennas, reflector antennas, radome design, and computational electromagnetics.
Vessen Vassilev received M.Sc degrees in "Radio Communications" from the Sofia Technical University in 1995, and in "Digital Communications" from Chalmers University of Technology in 1998. In 2003 he received his $\mathrm{PhD}$ degree from the department of "Radio and Space Science" at Chalmers University. Between 1998 and 2008 he has been working with the development of mm-wave receivers for applications in radio astronomy and space sciences. Instruments designed by him are currently in operation at the Atacama Pathfinder Experiment (APEX) telescope and at the Onsala Space Observatory. Since 2008 he has been with the Microwave Electronics Laboratory at the Department of Microtechnology and Nanoscience at Chalmers. His current interests are in the development mm-wavelength sensors based on MMIC technologies.

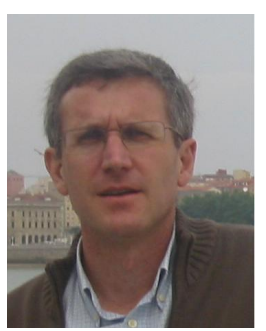

Mariano Baquero-Escudero was born in Murcia, Spain, on January 11, 1962. He received the degree in telecommunications engineering from the Polytechnic University of Catalonia (UPC), Barcelona, Spain, in 1986 and the Ph.D. degree from the Polytechnic University of Valencia (UPV), Valencia, Spain, in 1994. He became a Member (M) of IEEE in 1987. He was with the Antennas, Microwave and Radar Group, UPC, from 1986 to 1988, where he worked on the development of a cylindrical nearfield facility to measure a 3-D radar antenna in CESELSA. Since 1989, he has been with the UPV where he became a Full Professor in 2003. During 1995, he held a postdoctoral grant at the Joint Research Centre, European Commission, Ispra, Italy, where he developed highresolution algorithms for radar applications. From April 1996 to February 1998, he was a Vice-Dean of the Telecommunications Engineering School of Valencia. He is currently with the Communications Department and into the Institute of Telecommunications and Multimedia Application of the Polytechnic University of Valencia. His main research interests include microwave circuit and antenna analysis, design and measurement.

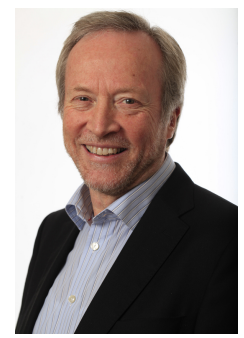

Per-Simon Kildal has been Professor at Chalmers University of Technology, Gothenburg, Sweden since 1989. He is now heading the Division of Antenna Systems at Department of Signals and Systems at Chalmers. Prof Kildal received two best paper awards for articles published in the IEEE Transactions on Antennas and Propagation, and he was the recipient of the 2011 Distinguished Achievements Award of the IEEE Antennas and Propagation Society. Kildal has authored an antenna textbook, and more than 150 journal articles and letters, most of them in IEEE or IET journals. He has designed two very large antennas, including the Gregorian dual-reflector feed of the Arecibo radiotelescope. He has invented several reflector antenna feeds, the latest being the so-called "Eleven antenna". Kildal is the originator of the concept of soft and hard surfaces, recently resulting in the gap waveguide, a new low-loss metamaterialbased transmission line advantageous in particular above $30 \mathrm{GHz}$. Kildal has received large individual grants from the Swedish research council VR and from the European Research Council ERC for research on gap waveguides. His research group has pioneered the reverberation chamber into an accurate measurement tool for antennas and wireless terminals subject to Rayleigh fading. This has been successfully commercialized in Bluetest AB. 